Homology, Homotopy and Applications, vol.10(3), 2008, pp.137-150

\title{
EXPLICIT FIBRANT REPLACEMENT FOR DISCRETE $G$-SPECTRA
}

\author{
DANIEL G. DAVIS \\ (communicated by Jack Morava)
}

\begin{abstract}
If $\mathcal{C}$ is the model category of simplicial presheaves on a site with enough points, with fibrations equal to the global fibrations, then it is well-known that the fibrant objects are, in general, mysterious. Thus, it is not surprising that, when $G$ is a profinite group, the fibrant objects in the model category of discrete $G$-spectra are also difficult to get a handle on. However, with simplicial presheaves, it is possible to construct an explicit fibrant model for an object in $\mathcal{C}$, under certain finiteness conditions. Similarly, in this paper, we show that if $G$ has finite virtual cohomological dimension and $X$ is a discrete $G$ spectrum, then there is an explicit fibrant model for $X$. Also, we give several applications of this concrete model related to closed subgroups of $G$.
\end{abstract}

\section{Introduction}

In this paper, $G$ always denotes a profinite group and by "spectrum" we mean a Bousfield-Friedlander spectrum of simplicial sets. In particular, a discrete G-spectrum is a $G$-spectrum such that each simplicial set $X_{k}$ is a simplicial object in the category of discrete $G$-sets (thus, the action map on the $l$-simplices,

$$
G \times\left(X_{k}\right)_{l} \rightarrow\left(X_{k}\right)_{l},
$$

is continuous when $\left(X_{k}\right)_{l}$ is regarded as a discrete space, for all $\left.l \geqslant 0\right)$. The category of discrete $G$-spectra, with morphisms being the $G$-equivariant maps of spectra, is denoted by $\mathrm{Spt}_{G}$.

As shown in [3, Section 3], $\mathrm{Spt}_{G}$ is a simplicial model category, where a morphism $f$ in $\mathrm{Spt}_{G}$ is a weak equivalence (cofibration) if and only if $f$ is a weak equivalence (cofibration) in Spt, the simplicial model category of spectra. Given $X \in \operatorname{Spt}_{G}$, the homotopy fixed point spectrum $X^{h G}$ is the total right derived functor of fixed points: $X^{h G}=\left(X_{f, G}\right)^{G}$, where $X \rightarrow X_{f, G}$ is a trivial cofibration and $X_{f, G}$ is fibrant, all in $\operatorname{Spt}_{G}$. This definition generalizes the classical definition of homotopy fixed point spectrum, in the case when $G$ is a finite group (see [3, pg. 337]).

Received December 31, 2007, revised March 30, 2008; published on December 5, 2008. 2000 Mathematics Subject Classification: 55P42, 55P91.

Key words and phrases: homotopy fixed points, discrete $G$-spectrum, homotopy limit.

This article is available at http://intlpress.com/HHA/v10/n3/a7

Copyright (c) 2008, International Press. Permission to copy for private use granted. 
Notice that we can loosen up the requirements on $X_{f, G}$. If $X \rightarrow X^{f}$ is a weak equivalence, with $X^{f}$ fibrant, all in $\mathrm{Spt}_{G}$, then, by the right lifting property of a fibrant object, there is a weak equivalence $X_{f, G} \rightarrow X^{f}$ in $\mathrm{Spt}_{G}$, so that

$$
X^{h G}=\left(X_{f, G}\right)^{G} \rightarrow\left(X^{f}\right)^{G}
$$

is a weak equivalence. Thus, we can identify $X^{h G}$ and $\left(X^{f}\right)^{G}$, and, hence, we only need a fibrant replacement $X^{f}$ to form $X^{h G}$. Henceforth, we relabel any such $X^{f}$ as $X_{f, G}$ and refer to it as a globally fibrant model for $X$. (Thus, from now on, $X \rightarrow X_{f, G}$ does not have to be a cofibration.)

The preceding discussion shows that a globally fibrant model $X_{f, G}$ is an important object. Of course, the model category axioms guarantee that $X_{f, G}$ always exists. But it is reasonable to ask for more. For example, in Spt, there is a functor

$$
Q: \mathrm{Spt} \rightarrow \mathrm{Spt}, \quad Z \mapsto Q(Z)=Z_{\mathrm{f}},
$$

where $Z_{\mathrm{f}}$ is a fibrant spectrum,

$$
\left(Z_{\mathrm{f}}\right)_{k}=\underset{n}{\operatorname{colim}} \Omega^{n}\left(\mathrm{Ex}^{\infty}\left(Z_{k+n}\right)\right)
$$

and there is a natural weak equivalence $Z \rightarrow Z_{\mathrm{f}}$ (see, for example, [14, pg. 524]). Hence, for the model category Spt, there is always an explicit model for fibrant replacement. Similarly, it is natural to wonder if an explicit model for $X_{f, G}$ is available.

But there is a difficulty with this. Let $G$-Sets $\mathbf{s}_{d f}$ be the Grothendieck site of finite discrete $G$-sets (e.g., see [10, Section 6.2]). There is an equivalence between Spt $_{G}$ and the category of sheaves of spectra on $G$-Sets $d f$ (the discrete $G$-spectrum $X$ corresponds to the sheaf of spectra $\operatorname{Hom}_{G}(-, X)$; see $[\mathbf{3}$, Section 3] for details), and it is well-known that, in general, for categories of simplicial presheaves, presheaves of spectra, and sheaves of spectra, there is no known explicit model for a globally fibrant object. In fact, the situation is such that [8, pg. 1049] says that "[t]he fibrant objects in all of these theories continue to be really quite mysterious." (A similar statement appears in [11], between Corollary 19 and Definition 20).

Nevertheless, under certain hypotheses, explicit models for globally fibrant objects are available in the cases of simplicial presheaves and presheaves of spectra. Such results are based on Jardine's result in [12, Proposition 3.3], which constructs an explicit globally fibrant model for a simplicial presheaf $P$ on the site ét $\left.\right|_{S}$, where $P$ and the scheme $S$ must satisfy certain finiteness conditions (and other hypotheses). For example, under similar finiteness conditions, [13, Proposition 3.20] follows the proof of Jardine's result to obtain a concrete globally fibrant model for a presheaf of spectra on a site with enough points.

Now suppose that $G$ has finite virtual cohomological dimension (see Definition 4.1) and that $X$ is a discrete $G$-spectrum. In this paper, we show that there is an explicit model for $X_{f, G}$, by expressing the homotopy limit for diagrams in $\mathrm{Spt}_{G}$ in terms of the homotopy limit for diagrams in Spt (see Theorem 2.3) and by modifying the proof of [3, Theorem 7.4] (which applies the two results cited above, [12, Proposition 3.3] and [13, Proposition 3.20]). We refer the reader to Theorem 4.2 for the precise statement of our main result; its formulation depends on definitions that are given in Section 3. 
Let $H$ be a closed subgroup of $G$. If $Y \rightarrow Z$ is a weak equivalence in $\operatorname{Spt}_{H}$, such that $Y$ is a globally fibrant model for $X$ in $\operatorname{Spt}_{G}$ and $Z$ is a globally fibrant model for $X$ in $\operatorname{Spt}_{H}$, then we label the map $Y \rightarrow Z$ as $r_{H}^{G}$. Note that the map

$$
X_{f, G} \rightarrow\left(X_{f, G}\right)_{f, H},
$$

a weak equivalence in $\mathrm{Spt}_{H}$, can be labelled as $r_{H}^{G}$, so that $r_{H}^{G}$ always exists. In Corollary 4.4, we show that the explicit globally fibrant model constructed in Theorem 4.2 yields an explicit model for $r_{H}^{G}$ (where, as before, we assume that $G$ has finite virtual cohomological dimension).

Section 5 explains that, when $H$ is a closed normal subgroup of $G$ and $X$ is a discrete $G$-spectrum, there are cases when $X^{h H}$, unlike $X^{H}$, is not known to be a $G / H$-spectrum. In Corollary 5.4, we point out that, if $G$ has finite virtual cohomological dimension, then Theorem 4.2 implies that $X^{h H}$ can always be taken to be a $G / H$-spectrum.

Throughout this paper, $U<_{o} G$ means that $U$ is an open subgroup of $G$.

\section{Acknowledgements}

I thank Mark Hovey for a helpful conversation. Also, I thank the referee for a helpful comment.

\section{Homotopy limits in the category of discrete $G$-spectra}

To explicitly construct the desired fibrant discrete $G$-spectrum, we first need to understand homotopy limits in the category of discrete $G$-spectra. Thus, we begin this section by following the presentation in $[\mathbf{9}]$ to give the general definition of the homotopy limit of a $\mathcal{C}$-diagram $X(-)$ in $\mathcal{M}$, where $\mathcal{M}$ is a simplicial model category and $X(-)$ is a diagram in $\mathcal{M}$ indexed by a small category $\mathcal{C}$.

Recall that, for a small category $\mathcal{C}$, the classifying space of $\mathcal{C}$ is the simplicial set $B \mathcal{C}$, with $l$-simplices $(B \mathcal{C})_{l}$ equal to the set of compositions

$$
c_{0} \stackrel{\sigma_{0}}{\longrightarrow} c_{1} \stackrel{\sigma_{1}}{\longrightarrow} \cdots \stackrel{\sigma_{l-1}}{\longrightarrow} c_{l}
$$

in $\mathcal{C}$ (see, for example, $[\mathbf{9}$, Definition 14.1.1] for the definition of the face and degeneracy maps).

Definition 2.1 ([9, Definition 18.1.8]). As above, let $\mathcal{M}$ be a simplicial model category and let $\mathcal{C}$ be a small category. Also, let $X=X(-)$ be a $\mathcal{C}$-diagram in $\mathcal{M}$; that is, $X$ is a functor $\mathcal{C} \rightarrow \mathcal{M}$, so that, for example, if $c \rightarrow d$ is a morphism in $\mathcal{C}$, then $X(c) \rightarrow X(d)$ is a morphism in $\mathcal{M}$. Then the homotopy limit of $X$ in $\mathcal{M}$, $\operatorname{holim}_{\mathcal{C}}^{\mathcal{M}} X$, is defined to be the equalizer of the diagram

$$
\prod_{c \in \mathcal{C}} X(c)^{B(\mathcal{C} \downarrow c)} \underset{\beta}{\stackrel{\alpha}{\longrightarrow}} \prod_{\sigma: c \rightarrow d} X(d)^{B(\mathcal{C} \downarrow c)},
$$

where the second product is indexed over all the morphisms in $\mathcal{C}$. Here, the map $\alpha$ is defined as follows: the projection of $\alpha$ onto the factor indexed by $\sigma: c_{0} \rightarrow c_{1}$ is equal 
to composing the projection

$$
\prod_{c \in \mathcal{C}} X(c)^{B(\mathcal{C} \downarrow c)} \rightarrow X\left(c_{0}\right)^{B\left(\mathcal{C} \downarrow c_{0}\right)}
$$

with the canonical map $X\left(c_{0}\right)^{B\left(\mathcal{C} \downarrow c_{0}\right)} \rightarrow X\left(c_{1}\right)^{B\left(\mathcal{C} \downarrow c_{0}\right)}$. The map $\beta$ is defined by letting the projection of $\beta$ onto the factor indexed by $\sigma$ be given by composing the projection

$$
\prod_{c \in \mathcal{C}} X(c)^{B(\mathcal{C} \downarrow c)} \rightarrow X\left(c_{1}\right)^{B\left(\mathcal{C} \downarrow c_{1}\right)}
$$

with the canonical map $X\left(c_{1}\right)^{B\left(\mathcal{C} \downarrow c_{1}\right)} \rightarrow X\left(c_{1}\right)^{B\left(\mathcal{C} \downarrow c_{0}\right)}$ that is induced by the map $B\left(\mathcal{C} \downarrow c_{0}\right) \rightarrow B\left(\mathcal{C} \downarrow c_{1}\right)$.

Remark 2.2. Note that the homotopy limit is an equalizer of a diagram involving products and cotensors, and, given a simplicial set $K$, the cotensor functor $(-)^{K}: \mathcal{M} \rightarrow \mathcal{M}$ is a right adjoint. Hence, the homotopy limit holim $\mathcal{C}_{\mathcal{M}}^{\mathcal{M}}(-)$ commutes with limits in $\mathcal{M}$.

If $X$ and $Z$ are $\mathcal{C}$-diagrams in $\mathrm{Spt}_{G}$ and $\mathrm{Spt}$, respectively, then we use the less cumbersome $\operatorname{holim}_{\mathcal{C}}^{G} X$ and $\operatorname{holim}_{\mathcal{C}} Z$ to denote $\operatorname{holim}_{\mathcal{C}}{ }^{\operatorname{Spt}_{G}} X$ and $\operatorname{holim}_{\mathcal{C}}^{\text {Spt }} Z$, respectively. As in Definition 2.1, given $c \in \mathcal{C}, X(c)$ is the object in $\mathcal{M}$ that is indexed by $c$ in the diagram $X$.

Theorem 2.3. If $X$ is a $\mathcal{C}$-diagram in $\mathrm{Spt}_{G}$, then there is an isomorphism

$$
\operatorname{holim}_{\mathcal{C}}{ }^{G} X \cong \underset{N \triangleleft \triangleleft_{o} G}{\operatorname{colim}}(\underset{\mathcal{C}}{\operatorname{holim}} X)^{N} \text {. }
$$

Proof. For each $c \in \mathcal{C}$, let $B_{c}$ denote the simplicial set $B(\mathcal{C} \downarrow c)$. Given a discrete $G$ spectrum $Y$ and a simplicial set $K$, let $\left(Y^{K}\right)_{G}$ and $Y^{K}$ denote the cotensor objects in $\mathrm{Spt}_{G}$ and Spt, respectively. Also, let $\prod^{G}$ and $\prod$ denote products in $\mathrm{Spt}_{G}$ and Spt, respectively. Then, by Definition $2.1, \operatorname{holim}_{\mathcal{C}}^{G} X$ is the equalizer of the diagram

$$
\prod_{c \in \mathcal{C}}^{G}\left(X(c)^{B_{c}}\right)_{G} \stackrel{\alpha}{\longrightarrow} \prod_{\sigma: c \rightarrow d}^{G}\left(X(d)^{B_{c}}\right)_{G}
$$

in $\mathrm{Spt}_{G}$.

To go further, we note how limits and cotensors in $\mathrm{Spt}_{G}$ are formed. Recall from [3, Remark 4.2] that, if $\left\{Y_{\alpha}\right\}_{\alpha}$ is any diagram in $\operatorname{Spt}_{G}$, then the limit of this diagram in the category $\operatorname{Spt}_{G}$ is $\operatorname{colim}_{N \triangleleft_{o} G}\left(\lim _{\alpha} Y_{\alpha}\right)^{N}$, where the limit in this expression is taken in the category of spectra. The colimit in this expression, and others like it, can be taken in the category of spectra, since the forgetful functor $\mathrm{Spt}_{G} \rightarrow \mathrm{Spt}$ is a left adjoint, by [3, Corollary 3.8]. Given a discrete $G$-spectrum $Y$ and a simplicial set $K$, the spectrum $Y^{K}$ can be regarded as a $G$-spectrum (but not necessarily a discrete $G$-spectrum), by using only the $G$-action on $Y$. Then

$$
\left(Y^{K}\right)_{G}=\operatorname{colim}_{N \triangleleft_{o} G}\left(Y^{K}\right)^{N}
$$

(e.g., see [6, (1.2.2)] and [12, pg. 42]). We apply these observations as follows.

First of all, note that $\operatorname{holim}_{\mathcal{C}}^{G} X$ is the equalizer in $\operatorname{Spt}_{G}$ of the diagram

$$
\operatorname{colim}_{N \triangleleft_{o} G}\left(\prod_{c \in \mathcal{C}}\left(X(c)^{B_{c}}\right)_{G}\right)^{N} \underset{\beta}{\stackrel{\alpha}{\longrightarrow}} \operatorname{colim}_{N \triangleleft_{o} G}\left(\prod_{\sigma: c \rightarrow d}\left(X(d)^{B_{c}}\right)_{G}\right)^{N} .
$$

Furthermore, let $S$ be a $G$-set (but not necessarily a discrete $G$-set) and let $U$ be 
an open normal subgroup of $G$. Then it is clear that $\left(\bigcup_{N \triangleleft_{G} G} S^{N}\right)^{U} \subset S^{U}$. Since $U \in\left\{N \mid N \triangleleft_{o} G\right\}, S^{U} \subset \bigcup_{N \triangleleft_{o} G} S^{N}$, and, hence, $S^{U} \subset\left(\bigcup_{N \triangleleft_{o} G} S^{N}\right)^{U}$. Thus, we can conclude that

$$
S^{U}=\left(\bigcup_{N \triangleleft_{o} G} S^{N}\right)^{U} .
$$

Similarly, if $Y$ is a $G$-spectrum,

$$
Y^{U} \cong\left(\operatorname{colim}_{N \triangleleft_{o} G} Y^{N}\right)^{U}
$$

Therefore,

$$
\left(\prod_{c \in \mathcal{C}}\left(X(c)^{B_{c}}\right)_{G}\right)^{U}=\prod_{c \in \mathcal{C}}\left(\operatorname{colim}_{N \triangleleft_{o} G}\left(X(c)^{B_{c}}\right)^{N}\right)^{U} \cong \prod_{c \in \mathcal{C}}\left(X(c)^{B_{c}}\right)^{U},
$$

and, similarly,

$$
\left(\prod_{\sigma: c \rightarrow d}\left(X(d)^{B_{c}}\right)_{G}\right)^{U} \cong \prod_{\sigma: c \rightarrow d}\left(X(d)^{B_{c}}\right)^{U} .
$$

The preceding two isomorphisms imply that $\operatorname{holim}_{\mathcal{C}}^{G} X$ is isomorphic to the equalizer in $\mathrm{Spt}_{G}$ of the diagram

$$
\underset{N \triangleleft_{o} G}{\operatorname{colim}}\left(\prod_{c \in \mathcal{C}} X(c)^{B_{c}}\right)^{N} \underset{\beta}{\stackrel{\alpha}{\longrightarrow}} \operatorname{colim}_{N \triangleleft_{o} G}\left(\prod_{\sigma: c \rightarrow d} X(d)^{B_{c}}\right)^{N} .
$$

Thus, $\operatorname{holim}_{\mathcal{C}}^{G} X \cong \operatorname{colim}_{N \triangleleft_{o} G} \mathcal{E}^{N}$, where $\mathcal{E}$ is the equalizer in Spt of the diagram

$$
\operatorname{colim}_{N \triangleleft_{o} G}\left(\prod_{c \in \mathcal{C}} X(c)^{B_{c}} \underset{\beta^{\prime}}{\stackrel{\alpha^{\prime}}{\longrightarrow}} \prod_{\sigma: c \rightarrow d} X(d)^{B_{c}}\right)^{N},
$$

where $\alpha^{\prime}$ and $\beta^{\prime}$ are the maps in the equalizer diagram for $\operatorname{holim}_{\mathcal{C}} X$.

Since filtered colimits and finite limits commute, $\mathcal{E} \cong \operatorname{colim}_{N \triangleleft_{o} G}\left(\mathcal{E}^{\prime}\right)^{N}$, where $\mathcal{E}^{\prime}$ is the equalizer in Spt of the diagram

$$
\prod_{c \in \mathcal{C}} X(c)^{B_{c}} \underset{\beta^{\prime}}{\stackrel{\alpha^{\prime}}{\longrightarrow}} \prod_{\sigma: c \rightarrow d} X(d)^{B_{c}} .
$$

Notice that $\mathcal{E}^{\prime}=\operatorname{holim}_{\mathcal{C}} X$.

If $U$ is an open normal subgroup of $G$, then $\left(\operatorname{holim}_{\mathcal{C}} X\right)^{U}$ is a $G / U$-spectrum, so that $\operatorname{colim}_{N \triangleleft_{o} G}\left(\operatorname{holim}_{\mathcal{C}} X\right)^{N}$ is a discrete $G$-spectrum. Also, given $Y \in \operatorname{Spt}_{G}$, there is an isomorphism $\operatorname{colim}_{N \triangleleft_{o} G} Y^{N} \cong Y$. Therefore, putting our various observations together, we obtain that

$$
\begin{aligned}
\operatorname{holim}_{\mathcal{C}} G X & \cong \operatorname{colim}_{N \triangleleft_{o} G} \mathcal{E}^{N} \cong \underset{N \triangleleft_{o} G}{\operatorname{colim}}\left(\underset{N^{\prime} \triangleleft_{o} G}{\operatorname{colim}}\left(\mathcal{E}^{\prime}\right)^{N^{\prime}}\right)^{N} \\
& \cong \operatorname{colim}_{N^{\prime} \triangleleft_{o} G}\left(\mathcal{E}^{\prime}\right)^{N^{\prime}}=\operatorname{colim}_{N \triangleleft_{o} G}(\operatorname{holim} X)^{N} .
\end{aligned}
$$

If $X$ is a $\mathcal{C}$-diagram of fibrant discrete $G$-spectra (that is, $X(c)$ is fibrant in $\operatorname{Spt}_{G}$, for all $c \in \mathcal{C}$ ), then holim $\mathcal{C}_{\mathcal{C}}^{G} X$ is a fibrant discrete $G$-spectrum, by $[\mathbf{9}$, Theorem 18.5.2 (2)], so that Theorem 2.3 gives the following result.

Corollary 2.4. If $X$ is a $\mathcal{C}$-diagram of fibrant discrete $G$-spectra, then the spectrum $\operatorname{colim}_{N \triangleleft_{o} G}\left(\operatorname{holim}_{\mathcal{C}} X\right)^{N}$ is a fibrant discrete $G$-spectrum. 
We conclude this section with some observations about Corollary 2.4.

Definition 2.5. If $P$ is a $\mathcal{C}$-diagram of presheaves of spectra on the site $G$-Sets $\mathbf{S}_{d f}$, then there is a presheaf of spectra $\operatorname{holim}_{\mathcal{C}} P$, defined by

$$
\underset{\mathcal{C}}{(\operatorname{holim}} P)(S)=\underset{\mathcal{C}}{\operatorname{holim}} P(S),
$$

for each $S \in G$-Sets $d f$.

Let $X$ be a $\mathcal{C}$-diagram in $\operatorname{Spt}_{G}$. Then it is natural to form the presheaf of spectra $\operatorname{holim}_{\mathcal{C}} \operatorname{Hom}_{G}(-, X)$. Also, let

$$
\mathcal{F}=\operatorname{Hom}_{G}\left(-, \underset{N \triangleleft_{o} G}{\operatorname{colim}}\left(\operatorname{holim}_{\mathcal{C}} X\right)^{N}\right),
$$

the canonical sheaf of spectra on the site $G$-Sets $_{d f}$ associated to the spectrum $\operatorname{colim}_{N \triangleleft_{o} G}\left(\operatorname{holim}_{\mathcal{C}} X\right)^{N}$ that is considered in Corollary 2.4.

The following lemma says that the presheaf $\operatorname{holim}_{\mathcal{C}} \operatorname{Hom}_{G}(-, X)$ is actually a sheaf of spectra, since it is isomorphic to $\mathcal{F}$.

Lemma 2.6. If $X$ is a $\mathcal{C}$-diagram of discrete $G$-spectra, then the presheaves of spectra $\mathcal{F}$ and $\operatorname{holim}_{\mathcal{C}} \operatorname{Hom}_{G}(-, X)$ on the site $G$-Sets $d f$ are isomorphic.

Proof. Let $S$ be a finite discrete $G$-set: $S$ can be identified with a disjoint union $\bigsqcup_{j=1}^{m} G / U_{j}$, where each $U_{j}$ is an open subgroup of $G$. Notice that the collection $\{N\}_{N \triangleleft_{o} G}$ of open normal subgroups of $G$ is a cofinal subcollection of the collection $\{U\}_{U<_{o} G}$ of open subgroups of $G$, so that, if $Y$ is a $G$-spectrum, there is an isomorphism $\operatorname{colim}_{N \triangleleft_{o} G} Y^{N} \cong \operatorname{colim}_{U<_{o} G} Y^{U}$ of $G$-spectra. Hence,

$$
\begin{aligned}
\mathcal{F}(S) & \cong \prod_{j=1}^{m}\left(\operatorname{colim}_{N \triangleleft_{o} G}(\underset{\mathcal{C}}{\operatorname{holim}} X)^{N}\right)^{U_{j}} \\
& \cong \prod_{j=1}^{m}\left(\operatorname{colim}_{U<_{o} G}(\underset{\mathcal{C}}{\operatorname{holim}} X)^{U}\right)^{U_{j}} \\
& \cong \prod_{j=1}^{m}\left(\underset{\mathcal{C}}{\operatorname{holim} X)^{U_{j}}}\right. \\
& \cong \operatorname{holim}_{\mathcal{C}} \operatorname{Hom}_{G}(S, X),
\end{aligned}
$$

where the third isomorphism is due to the fact that $U_{j} \in\left\{U \mid U<_{o} G\right\}$ (as in the proof of Theorem 2.3) and the last isomorphism applies Remark 2.2. This chain of isomorphisms shows that there is an isomorphism $\mathcal{F}(S) \cong \operatorname{holim}_{\mathcal{C}} \operatorname{Hom}_{G}(S, X)$ that is natural for $S \in G$-Sets $d f$, so that $\mathcal{F}$ and $\operatorname{holim}_{\mathcal{C}} \operatorname{Hom}_{G}(-, X)$ are isomorphic presheaves of spectra.

Remark 2.7. Let $X$ be a $\mathcal{C}$-diagram of fibrant discrete $G$-spectra. Then the assertion of Corollary 2.4 that $\operatorname{colim}_{N \triangleleft_{o} G}\left(\operatorname{holim}_{\mathcal{C}} X\right)^{N}$ is a fibrant discrete $G$-spectrum is equivalent to claiming that $\mathcal{F}$ is a globally fibrant presheaf of spectra (see [3, pg. 333]). Also, by Lemma 2.6, to show that $\mathcal{F}$ is globally fibrant, it suffices to show that $\operatorname{holim}_{\mathcal{C}} \operatorname{Hom}_{G}(-, X)$ is a globally fibrant presheaf. This can be done by adapting $[\mathbf{1 2}$, Proposition 3.3] and [3, Lemma 7.3], since $\operatorname{Hom}_{G}(-, X)$ is a $\mathcal{C}$-diagram of globally fibrant presheaves of spectra. This gives a somewhat different way of obtaining Corollary 2.4 . 


\section{The explicit construction of a fibrant discrete $G$-spectrum}

In this section, we use Corollary 2.4 to construct the fibrant object in $\mathrm{Spt}_{G}$ that is our primary object of interest. We begin with several definitions that are standard in the theory of discrete $G$-modules and discrete $G$-spectra.

Definition 3.1. If $A$ is an abelian group with the discrete topology, let $\operatorname{Map}_{c}(G, A)$ be the abelian group of continuous maps from $G$ to $A$. If $Z$ is a spectrum, then one can also define the discrete $G$-spectrum $\operatorname{Map}_{c}(G, Z)$, where the $l$-simplices $\left(\operatorname{Map}_{c}(G, Z)_{k}\right)_{l}$ of the $k$ th simplicial set of the $\operatorname{spectrum}_{\operatorname{Map}_{c}}(G, Z)$ are given by $\operatorname{Map}_{c}\left(G,\left(Z_{k}\right)_{l}\right)$, the set of continuous maps from $G$ to $\left(Z_{k}\right)_{l}$. Here, $\left(Z_{k}\right)_{l}$ is given the discrete topology and the $G$-action on $\operatorname{Map}_{c}(G, Z)$ is induced on the level of sets by $(g \cdot f)\left(g^{\prime}\right)=f\left(g^{\prime} g\right)$, for $g, g^{\prime} \in G$ and $f \in \operatorname{Map}_{c}\left(G,\left(Z_{k}\right)_{l}\right)$, for each $k, l \geqslant 0$. This action also makes $\operatorname{Map}_{c}(G, A)$ a discrete $G$-module.

Definition 3.2. Consider the functor

$$
\Gamma_{G}: \operatorname{Spt}_{G} \rightarrow \operatorname{Spt}_{G}, \quad X \mapsto \Gamma_{G}(X)=\operatorname{Map}_{c}(G, X),
$$

where $\Gamma_{G}(X)$ has the $G$-action given by Definition 3.1. As explained in [3, Definition 7.1], the functor $\Gamma_{G}$ forms a triple and there is a cosimplicial discrete $G$-spectrum $\Gamma_{G}^{\bullet} X$, where, for all $n \geqslant 0$,

$$
\left(\Gamma_{G}^{\bullet} X\right)^{n} \cong \operatorname{Map}_{c}\left(G^{n+1}, X\right)
$$

Here, the spectrum $\operatorname{Map}_{c}\left(G^{n+1}, X\right)$ is defined as in Definition 3.1, since the cartesian product $G^{n+1}$ is a profinite group, and its discrete $G$-action is given by the $G$-action on the constituent sets that is given by

$$
(g \cdot f)\left(g_{1}, g_{2}, g_{3}, \ldots, g_{n+1}\right)=f\left(g_{1} g, g_{2}, g_{3}, \ldots, g_{n+1}\right) .
$$

The next definition restates Definition 3.2 in the context of discrete $G$-modules.

Definition 3.3. Let $\operatorname{DMod}(G)$ be the category of discrete $G$-modules. Then, as in Definition 3.2, there is a functor

$$
\Gamma_{G}: \operatorname{DMod}(G) \rightarrow \operatorname{DMod}(G), \quad M \mapsto \Gamma_{G}(M)=\operatorname{Map}_{c}(G, M),
$$

and, given a discrete $G$-module $M$, there is a cosimplicial discrete $G$-module $\Gamma_{G}^{\bullet} M$.

Definition 3.4 ([3, Remark 7.5]). Given a discrete $G$-spectrum $X$, let

$$
\widehat{X}=\underset{N \triangleleft_{o} G}{\operatorname{colim}}\left(X^{N}\right)_{\mathrm{f}} .
$$

Notice that $\widehat{X}$ is a discrete $G$-spectrum, since functorial fibrant replacement in Spt (see the introduction) implies that each $\left(X^{N}\right)_{\mathrm{f}}$ is a $G / N$-spectrum. Also, $\widehat{X}$ is fibrant as a spectrum and there is a weak equivalence

$$
\psi: X \cong \operatorname{colim}_{N \triangleleft_{o} G} X^{N} \rightarrow \operatorname{colim}_{N \triangleleft_{o} G}\left(X^{N}\right)_{\mathrm{f}}=\widehat{X}
$$

that is $G$-equivariant.

We define some useful terminology. If $X^{\bullet}$ is a cosimplicial object in $\mathrm{Spt}_{G}$, then $X^{\bullet}$ is a cosimplicial discrete $G$-spectrum. If $X^{\bullet}$ is a cosimplicial discrete $G$-spectrum 
such that $X^{n}$ is a fibrant discrete $G$-spectrum, for all $n \geqslant 0$, then $X^{\bullet}$ is a cosimplicial fibrant discrete $G$-spectrum.

The following result defines the explicit globally fibrant object that is of particular interest to us.

Theorem 3.5. Let $G$ be a profinite group, and let $H$ be a closed subgroup of $G$. If $X$ is a discrete $G$-spectrum, then the discrete $H$-spectrum

$$
\underset{K \triangleleft_{o} H}{\operatorname{colim}}\left(\underset{\Delta}{\operatorname{holim}} \Gamma_{G}^{\bullet} \widehat{X}\right)^{K}
$$

is fibrant in the model category of discrete $H$-spectra. In particular, the discrete $G$ spectrum

$$
\underset{N \triangleleft_{o} G}{\operatorname{colim}}\left(\operatorname{holim} \Gamma_{G}^{\bullet} \widehat{X}\right)^{N}
$$

is fibrant in $\mathrm{Spt}_{G}$.

Proof. By Corollary 2.4, we only need to show that $\Gamma_{G}^{\bullet} \widehat{X}$ is a cosimplicial fibrant discrete $H$-spectrum. By [15, Proposition 1.3.4 (c)], there is a homeomorphism

$$
h: H \times G / H \rightarrow G
$$

that is $H$-equivariant, where $H$ acts on the source by acting only on the factor $H$ and $G / H$ is the profinite space $G / H \cong \lim _{N \triangleleft_{o} G} G / N H$.

Given a spectrum $Z$ and a profinite space $W=\lim _{\alpha} W_{\alpha}$, where each $W_{\alpha}$ is a finite

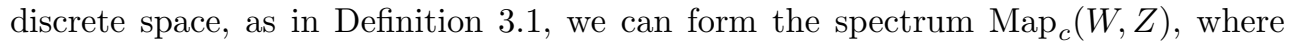
$\left(\operatorname{Map}_{c}(W, Z)_{k}\right)_{l}=\operatorname{Map}_{c}\left(W,\left(Z_{k}\right)_{l}\right)$, and there is an isomorphism

$$
\operatorname{Map}_{c}(W, Z) \cong \operatorname{colim}_{\alpha} \prod_{w \in W_{\alpha}} Z \text {. }
$$

Thus,

$$
\operatorname{Map}_{c}(G / H, \widehat{X}) \cong \operatorname{colim}_{N \triangleleft_{o} G} \prod_{G / N H} \widehat{X} .
$$

Since filtered colimits commute with finite limits,

$$
\prod_{G / N H} \widehat{X} \cong \prod_{G / N H} \operatorname{colim}_{N^{\prime} \triangleleft_{o} G}(\widehat{X})^{N^{\prime}} \cong \operatorname{colim}_{N^{\prime} \triangleleft_{o} G}\left(\prod_{G / N H} \widehat{X}\right)^{N^{\prime}},
$$

and it follows that $\operatorname{Map}_{c}(G / H, \widehat{X})$ is a discrete $G$-spectrum, with $G$ acting only on $\widehat{X}$. Therefore, by applying the homeomorphism $h$, there is an isomorphism

$$
\operatorname{Map}_{c}(G, \widehat{X}) \cong \operatorname{Map}_{c}\left(H, \operatorname{Map}_{c}(G / H, \widehat{X})\right)
$$

of discrete $H$-spectra.

Recall from [3, Corollary 3.8] that if $Z$ is a fibrant spectrum, then $\operatorname{Map}_{c}(H, Z)$ is a fibrant discrete $H$-spectrum. Also, since $\widehat{X}$ is a fibrant spectrum, the product $\prod_{G / N H} \widehat{X}$ is also a fibrant spectrum, so that, by $(1), \operatorname{Map}_{c}(G / H, \widehat{X})$ is fibrant in Spt. Then, by applying these observations to (2), we obtain that $\operatorname{Map}_{c}(G, \widehat{X})$ is a fibrant discrete $H$-spectrum. Hence, $\operatorname{Map}_{c}(G, \widehat{X})$ is a fibrant spectrum, by [3, Lemma 3.10], so that $\operatorname{Map}_{c}\left(G, \operatorname{Map}_{c}(G, \widehat{X})\right)$ is a fibrant discrete $H$-spectrum, by applying the previous argument again. Thus, iteration of this argument shows that $\Gamma_{G}^{\bullet} \widehat{X}$ is a cosimplicial fibrant discrete $H$-spectrum. 


\section{Completing the proof of the main result}

In this section, we finish the proof of the main result. Also, we discuss several consequences of having a concrete model for $X_{f, G}$, given a discrete $G$-spectrum $X$.

Definition 4.1. Let $H_{c}^{*}(G ; M)$ denote the continuous cohomology of $G$ with coefficients in the discrete $G$-module $M$. Then a profinite group $G$ has finite virtual cohomological dimension (or finite vcd) if there exists an open subgroup $H$ of $G$ and a non-negative integer $m$, such that $H_{c}^{s}(H ; M)=0$, for all discrete $H$-modules $M$ and all $s \geqslant m$.

Many of the profinite groups that one works with, in practice, have finite vcd. For example, if $G$ is a compact $p$-adic analytic group, then $G$ has finite vcd (see the discussion in [3, pg. 330]).

Let $X$ be a discrete $G$-spectrum. Then there is a $G$-equivariant monomorphism $i: X \rightarrow \operatorname{Map}_{c}(G, X)$ that is defined, on the level of sets, by $i(x)(g)=g \cdot x$. Then $i$ induces a map $X \rightarrow \Gamma_{G}^{\bullet} X$ of cosimplicial discrete $G$-spectra, where, here, $X$ is the constant diagram. Thus, the composition

$$
X \stackrel{\psi}{\rightarrow} \widehat{X} \stackrel{\cong}{\rightrightarrows} \lim _{\Delta} \widehat{X} \rightarrow \operatorname{holim}_{\Delta} \widehat{X} \rightarrow \operatorname{holim}_{\Delta} \Gamma_{G}^{\bullet} \widehat{X}
$$

of canonical maps defines the $G$-equivariant map

$$
\widehat{\psi}: X \rightarrow \operatorname{holim}_{\Delta} \Gamma_{G}^{\bullet} \widehat{X}
$$

(the canonical map $\lim _{\Delta} \widehat{X} \rightarrow \operatorname{holim}_{\Delta} \widehat{X}$ is defined in [9, Example 18.3.8 (2)]).

Note that there is a homotopy spectral sequence

$$
E_{2}^{s, t}=\pi^{s}\left(\pi_{t}\left(\Gamma_{G}^{\bullet} \widehat{X}\right)\right) \Rightarrow \pi_{t-s}\left(\underset{\Delta}{\operatorname{holim}} \Gamma_{G}^{\bullet} \widehat{X}\right),
$$

where $E_{2}^{0, t} \cong \pi_{t}(X)$ and $E_{2}^{s, t}=0$, when $s>0$, by [3, Section 7]. Thus, the spectral sequence collapses, so that the map $\widehat{\psi}$ is a weak equivalence.

Now let $H$ be a closed subgroup of $G$. Then $X$ is a discrete $H$-spectrum, so that $X \cong \operatorname{colim}_{K \triangleleft_{o} H} X^{K}$. Composing this isomorphism with the map $\operatorname{colim}_{K \triangleleft_{o} H}(\widehat{\psi})^{K}$ gives the $H$-equivariant map

$$
\Psi: X \rightarrow \underset{K \triangleleft_{o} H}{\operatorname{colim}}\left(\operatorname{holim}_{\Delta} \Gamma_{G}^{\bullet} \widehat{X}\right)^{K} .
$$

Now we show that if $G$ has finite vcd, then $\Psi$ is a weak equivalence. As mentioned in the introduction, the proof (below) closely follows the proof of [3, Theorem 7.4], so that our proof will be somewhat abbreviated. Also, we should mention that the proof of [3, Theorem 7.4] follows the arguments given in [12, proof of Proposition 3.3] and [13, Proposition 3.20].

Theorem 4.2. Let $G$ have finite vcd, let $X$ be a discrete $G$-spectrum, and let $H$ be a closed subgroup of $G$. Then the map

$$
\Psi: X \rightarrow \operatorname{colim}_{K \triangleleft_{o} H}\left(\operatorname{holim}_{\Delta} \Gamma_{G}^{\bullet} \widehat{X}\right)^{K}
$$

is a weak equivalence in the category of discrete $H$-spectra, such that the target is a fibrant discrete $H$-spectrum. 
Proof. Because of the earlier Theorem 3.5, we only have to prove that $\Psi$ is a weak equivalence of spectra.

Since $H$ is closed in $G, H$ also has finite vcd. Hence, $H$ has a collection $\{U\}$ of open normal subgroups such that: (a) $\{U\}$ is a cofinal subcollection of $\{K\}_{K \triangleleft_{o} H}$ (so, for example, $H \cong \lim _{U} H / U$ ), and $(b)$ for all $U, H_{c}^{s}(U ; M)=0$, for all $s \geqslant m$, where $m$ is some natural number that is independent of $U$, and for all discrete $U$-modules $M$. Thus,

$$
\underset{K \triangleleft_{o} H}{\operatorname{colim}}\left(\operatorname{holim}_{\Delta} \Gamma_{G}^{\bullet} \widehat{X}\right)^{K} \cong \operatorname{colim}_{U}\left(\operatorname{holim}_{\Delta} \Gamma_{G}^{\bullet} \widehat{X}\right)^{U},
$$

so that, to show that $\Psi$ is a weak equivalence, it suffices to show that the map

$$
\widehat{\Psi}: X \rightarrow \underset{U}{\operatorname{colim}}\left(\operatorname{holim}_{\Delta} \Gamma_{G}^{\bullet} \widehat{X}\right)^{U} \cong \operatorname{colim}_{U} \operatorname{holim}_{\Delta}\left(\Gamma_{G}^{\bullet} \widehat{X}\right)^{U},
$$

induced by $\Psi$ and (3), is a weak equivalence.

Notice that each $U$ is a closed subgroup of $G$. Then, for each $U,\left(\Gamma_{G}^{\bullet} \widehat{X}\right)^{U}$ is a cosimplicial fibrant spectrum, so that there is a conditionally convergent homotopy spectral sequence

$$
E_{2}^{s, t}(U)=\pi^{s} \pi_{t}\left(\left(\Gamma_{G}^{\bullet} \widehat{X}\right)^{U}\right) \Rightarrow \pi_{t-s}\left(\operatorname{holim}_{\Delta}\left(\Gamma_{G}^{\bullet} \widehat{X}\right)^{U}\right),
$$

with

$$
E_{2}^{s, t}(U) \cong H_{c}^{s}\left(U ; \pi_{t}(X)\right)
$$

(these assertions are verified in the proof of [3, Lemma 7.12]).

Since $E_{2}^{s, *}(U)=0$ whenever $s \geqslant m$, the $E_{2}$-terms $E_{2}^{*, *}(U)$ are uniformly bounded on the right. Therefore, by [13, Proposition 3.3], taking a colimit over $\{U\}$ of the spectral sequences in (4) gives the spectral sequence

$$
E_{2}^{s, t}=\operatorname{colim}_{U} H_{c}^{s}\left(U ; \pi_{t}(X)\right) \Rightarrow \pi_{t-s}\left(\operatorname{colim}_{U} \operatorname{holim}_{\Delta}\left(\Gamma_{G}^{\bullet} \widehat{X}\right)^{U}\right) .
$$

Notice that

$$
E_{2}^{*, t} \cong H_{c}^{*}\left(\lim _{U} U ; \pi_{t}(X)\right) \cong H_{c}^{*}\left(\{e\} ; \pi_{t}(X)\right),
$$

which is isomorphic to $\pi_{t}(X)$, concentrated in degree zero. Thus, spectral sequence (5) collapses, so that, for all $t, \pi_{t}\left(\operatorname{colim}_{U} \operatorname{holim}_{\Delta}\left(\Gamma_{G}^{\bullet} \widehat{X}\right)^{U}\right) \cong \pi_{t}(X)$, and, hence, $\widehat{\Psi}$ is a weak equivalence.

Let $X$ be a $\mathcal{C}$-diagram of discrete $G$-spectra, where $\mathcal{C}$ is a small category. Then, by Theorem 2.3, there is a canonical map

$$
\phi(X, G): \operatorname{holim}_{\mathcal{C}}^{G} X \cong \operatorname{colim}_{N \triangleleft_{o} G}\left(\operatorname{holim}_{\mathcal{C}} X\right)^{N} \rightarrow \operatorname{holim}_{\mathcal{C}} X
$$

that is $G$-equivariant.

Corollary 4.3. Let $G$ have finite vcd, let $X$ be a discrete $G$-spectrum, and let $H$ be a closed subgroup of $G$. Then the $H$-equivariant map

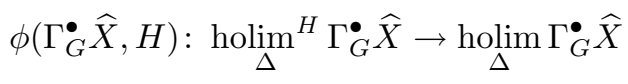

is a weak equivalence in Spt. 
Proof. Notice that $\widehat{\psi}=\phi\left(\Gamma_{G}^{\bullet} \widehat{X}, H\right) \circ \Psi$. Then the desired conclusion follows from the fact that $\widehat{\psi}$ and $\Psi$ are weak equivalences, where the latter fact is from Theorem 4.2.

In the introduction, we pointed out that a weak equivalence $r_{H}^{G}: X_{f, G} \rightarrow X_{f, H}$ in $\mathrm{Spt}_{H}$ always exists. The following result uses Theorem 4.2 to give a concrete model for $r_{H}^{G}$.

Corollary 4.4. Let $G$ have finite vcd, let $X$ be a discrete $G$-spectrum, and let $H$ be a closed subgroup of $G$. Then there is a weak equivalence

$$
r_{H}^{G}: \operatorname{colim}_{N \triangleleft_{o} G}\left(\operatorname{holim}_{\Delta} \Gamma_{G}^{\bullet} \widehat{X}\right)^{N} \rightarrow \underset{K \triangleleft_{o} H}{\operatorname{colim}}\left(\operatorname{holim}_{\Delta} \Gamma_{G}^{\bullet} \widehat{X}\right)^{K}
$$

in $\mathrm{Spt}_{H}$, where the source of this map is a fibrant discrete G-spectrum and the target is a fibrant discrete $H$-spectrum.

Proof. Let $N$ be an open normal subgroup of $G$. Then $N \cap H$ is an open normal subgroup of $H$ and, hence, there is a canonical map

$$
\left(\underset{\Delta}{\operatorname{holim}} \Gamma_{G}^{\bullet} \widehat{X}\right)^{N} \hookrightarrow\left(\operatorname{holim}_{\Delta} \Gamma_{G}^{\bullet} \widehat{X}\right)^{N \cap H} \rightarrow \underset{K \triangleleft_{o} H}{\operatorname{colim}}\left(\operatorname{holim}_{\Delta} \Gamma_{G}^{\bullet} \widehat{X}\right)^{K} .
$$

These maps, as $N$ varies, induce the desired map, which is easily seen to be $H$ equivariant. In $\operatorname{Spt}_{H}$, the weak equivalence $X \rightarrow \operatorname{colim}_{K \triangleleft_{o} H}\left(\operatorname{holim}_{\Delta} \Gamma_{G}^{\bullet} \widehat{X}\right)^{K}$ is the composition of the weak equivalence $X \rightarrow \operatorname{colim}_{N \triangleleft_{o} G}\left(\operatorname{holim}_{\Delta} \Gamma_{G}^{\bullet} \widehat{X}\right)^{N}$ and $r_{H}^{G}$, so that $r_{H}^{G}$ is a weak equivalence.

The following result is a special case of the fact that, if $H$ is open in $G$, then a fibrant discrete $G$-spectrum is also fibrant as a discrete $H$-spectrum (see [4, Lemma $3.1]$ and [10, Remark 6.26]).

Corollary 4.5. Let $G$ have finite vcd and let $X$ be a discrete $G$-spectrum. If $H$ is an open subgroup of $G$, then $\operatorname{colim}_{N \triangleleft_{o} G}\left(\operatorname{holim}_{\Delta}\left(\Gamma_{G}^{\bullet} \widehat{X}\right)\right)^{N}$, a fibrant discrete $G$-spectrum, is also a fibrant discrete $H$-spectrum.

Proof. By Theorem 4.2, the spectrum $\operatorname{colim}_{K \triangleleft_{o} H}\left(\operatorname{holim}_{\Delta}\left(\Gamma_{G}^{\bullet} \widehat{X}\right)\right)^{K}$ is a fibrant discrete $H$-spectrum. Thus, to verify the corollary, it suffices to show that this fibrant discrete $H$-spectrum is isomorphic to $\operatorname{colim}_{N \triangleleft_{o} G}\left(\operatorname{holim}_{\Delta}\left(\Gamma_{G}^{\bullet} \widehat{X}\right)\right)^{N}$ in $\operatorname{Spt}_{H}$.

Note that if $U$ is an open subgroup of $H$, then $U$ is also an open subgroup of $G$, so that

$$
\left\{H \cap V \mid V<_{o} G\right\}=\left\{U \mid U<_{o} H\right\} .
$$

Also, the sets $\left\{H \cap V \mid V<_{o} G\right\}$ and $\left\{N \mid N \triangleleft_{o} G\right\}$ are cofinal subcollections of the set $\left\{V \mid V<_{o} G\right\}$, so that

$$
\begin{aligned}
\underset{N \triangleleft_{o} G}{\operatorname{colim}}\left(\operatorname{holim}_{\Delta}\left(\Gamma_{G}^{\bullet} \widehat{X}\right)\right)^{N} & \cong \underset{V<_{o} G}{\operatorname{colim}}\left(\operatorname{holim}_{\Delta}\left(\Gamma_{G}^{\bullet} \widehat{X}\right)\right)^{V} \\
& \cong \operatorname{colim}_{V<_{o} G}\left(\operatorname{holim}\left(\Gamma_{G}^{\bullet} \widehat{X}\right)\right)^{H \cap V} \\
& =\underset{U<_{o} H}{\operatorname{colim}}\left(\operatorname{holim}_{\Delta}\left(\Gamma_{G}^{\bullet} \widehat{X}\right)\right)^{U} \\
& \cong \underset{K \triangleleft_{o} H}{\operatorname{colim}_{\Delta}}\left(\operatorname{holim}_{\Delta}\left(\Gamma_{G}^{\bullet} \widehat{X}\right)\right)^{K} .
\end{aligned}
$$




\section{Understanding $X^{h H}$ when $H$ is closed, and not open, in $G$}

In this section, we use the explicit fibrant model of Theorem 4.2 to improve (somewhat) our understanding of $X^{h H}$, for $X \in \mathrm{Spt}_{G}$, when $G$ has finite vcd and $H$ is a closed non-open subgroup of $G$.

In the introduction, we pointed out that if $L$ is any profinite group, $Z \in \mathrm{Spt}_{L}$, $Z \rightarrow Z_{f, L}$ is a trivial cofibration, and $Z \rightarrow Z^{f, L}$ is a weak equivalence, with $Z_{f, L}$ and $Z^{f, L}$ fibrant, all in $\mathrm{Spt}_{L}$, then $Z^{h L}=\left(Z_{f, L}\right)^{L} \rightarrow\left(Z^{f, L}\right)^{L}$ is a weak equivalence, so that we can identify $Z^{h L}$ and $\left(Z^{f, L}\right)^{L}$. For the upcoming discussion, we make this identification explicit.

Definition 5.1. If $L$ is a profinite group and $Z$ is a discrete $L$-spectrum, we define $Z^{h L}=\left(Z_{f, L}\right)^{L}$, where $Z \rightarrow Z_{f, L}$ is a weak equivalence and $Z_{f, L}$ is fibrant, all in $\operatorname{Spt}_{L}$.

Theorem 5.2. If $G$ has finite vcd, $H$ is a closed subgroup of $G$, and $X$ is a discrete $G$-spectrum, then

$$
X^{h H} \cong\left(\underset{\Delta}{\operatorname{holim}}\left(\Gamma_{G}^{\bullet} \widehat{X}\right)\right)^{H}
$$

Proof. By Definition 5.1 and Theorem 4.2,

$$
X^{h H}=\left(\operatorname{colim}_{K \triangleleft_{o} H}\left(\operatorname{holim}_{\Delta}\left(\Gamma_{G}^{\bullet} \widehat{X}\right)\right)^{K}\right)^{H} .
$$

As in the proof of Theorem 2.3, since $H$ is an open normal subgroup of itself, this expression simplifies to give the desired result.

Remark 5.3. Suppose that the hypotheses of Theorem 5.2 are satisfied. In [3, Remark 7.13], by using a different argument, we noted that there are certain weak equivalences that permit $\left(\operatorname{holim}_{\Delta}\left(\Gamma_{G}^{\bullet} \widehat{X}\right)\right)^{H}$ to be taken as a definition of $X^{h H}$. (To be precise, $[\mathbf{3}$, Remark 7.13] uses " $X_{f, G}$ " instead of $\widehat{X}$ in the expression $\left(\operatorname{holim}_{\Delta}\left(\Gamma_{G}^{\bullet} \widehat{X}\right)\right)^{H}$, but it is not hard to see that the remark is still valid with $\widehat{X}$ in place of " $X_{f, G}$.") However, Theorem 5.2 puts this definition on firmer ground by showing that it comes from taking the $H$-fixed points of a fibrant replacement for $X$.

For this paragraph and the next, let $X$ be a discrete $G$-spectrum and let $H$ be a closed proper normal subgroup of $G$, such that $H \neq\{e\}$. Then $G / H$ is a profinite group and the $H$-fixed point spectrum $X^{H}$ is a $G / H$-spectrum. However, as noted in [4, Sections 1 and 3], the corresponding situation is more complicated with $\mathrm{H}$ homotopy fixed points. It is natural to expect $X^{h H}$ to be a $G / H$-spectrum, but, given an arbitrary fibrant replacement $X_{f, H}$ (as guaranteed by the model category axioms), in general, there is no reason to assume that $X_{f, H}$ carries a $G$-action, so that $\left(X_{f, H}\right)^{H}$ need not be a $G / H$-spectrum. For example, if $H$ has finite vcd, then $X_{f, H}$ can be taken to be $\operatorname{colim}_{K \triangleleft_{o} H}\left(\operatorname{holim}_{\Delta}\left(\Gamma_{H}^{\bullet} \widehat{X}\right)\right)^{K}$, a discrete $H$-spectrum that is a $G$-spectrum only when additional hypotheses are satisfied.

If $H$ is open in $G$, then, as recalled at the end of the preceding section, $X_{f, G}$ is a fibrant discrete $H$-spectrum, so that $X^{h H}=\left(X_{f, G}\right)^{H}$ is easily seen to be a $G / H$-spectrum. However, if $H$ is not open in $G$, then the situation is much more 
complicated: it is natural to wonder if the map

$$
\left(r_{H}^{G}\right)^{H}:\left(X_{f, G}\right)^{H} \rightarrow\left(X_{f, H}\right)^{H}=X^{h H}
$$

is a weak equivalence, but the evidence indicates that it does not have to be (even when $G$ has finite vcd), since, for example, $X_{f, G}$ is not known to be fibrant in $\mathrm{Spt}_{H}$. (There is no example known of $\left(r_{H}^{G}\right)^{H}$ failing to be a weak equivalence, but there are several arguments indicating that there should be such examples; see [4, Sections 1 , 3 , and 4] and [1, Sections 3.5 and 3.6] for more discussion on this issue.) Thus, the theory of homotopy fixed points is faced with the undesirable fact that, in general, when $H$ is not open in $G$, it is not known how to show that $X^{h H}$ is a $G / H$-spectrum. However, Theorem 5.2 immediately implies the following result, for the case when $G$ has finite vcd.

Corollary 5.4. If $G$ has finite vcd, with $H$ a closed normal subgroup of $G$, and $X$ is a discrete $G$-spectrum, then $X^{h H}$, when taken to be

$$
X^{h H} \cong\left(\underset{\Delta}{\operatorname{holim}}\left(\Gamma_{G}^{\bullet} \widehat{X}\right)\right)^{H}
$$

is a $G / H$-spectrum.

In the context of Corollary 5.4, it is natural to inquire about the $G / H$-homotopy fixed points of $X^{h H}$. But there are non-trivial issues involved here; we refer the reader to $[4]$ for more on this topic.

\section{References}

[1] M. Behrens and D.G. Davis, The homotopy fixed point spectra of profinite Galois extensions, submitted for publication, arXiv:math.AT/0808.1092.

[2] A.K. Bousfield and D.M. Kan, Homotopy limits, completions and localizations, Lecture Notes in Mathematics 304, Springer-Verlag, New York, 1972.

[3] D.G. Davis, Homotopy fixed points for $L_{K(n)}\left(E_{n} \wedge X\right)$ using the continuous action, J. Pure Appl. Algebra 206 (2006), 322-354.

[4] D.G. Davis, Iterated homotopy fixed points for the Lubin-Tate spectrum, submitted for publication, arXiv:math.AT/0610907.

[5] E.S. Devinatz and M.J. Hopkins, Homotopy fixed point spectra for closed subgroups of the Morava stabilizer groups, Topology 43 (2004), 1-47.

[6] P.G. Goerss, Homotopy fixed points for Galois groups, in The Čech Centennial (Boston, MA, 1993), pages 187-224, Amer. Math. Soc., Providence, RI, 1995.

[7] P.G. Goerss and J.F. Jardine, Simplicial homotopy theory, Birkhäuser Verlag, Basel, 1999.

[8] P.G. Goerss and J.F. Jardine, Localization theories for simplicial presheaves, Canad. J. Math. 50 (1998), 1048-1089.

[9] P.S. Hirschhorn, Model categories and their localizations, Mathematical Surveys and Monographs 99, Amer. Math. Soc., Providence, RI, 2003.

[10] J.F. Jardine, Generalized étale cohomology theories, Progress in Mathematics 146, Birkhäuser Verlag, Basel, 1997. 
[11] J.F. Jardine, Generalised sheaf cohomology theories, in Axiomatic, enriched and motivic homotopy theory, NATO Sci. Ser. II Math. Phys. Chem. 131, pages 29-68, Kluwer, Acad. Publ., Dordrecht, 2004.

[12] J.F. Jardine, Simplicial presheaves, J. Pure Appl. Algebra 47 (1987), 35-87.

[13] S.A. Mitchell, Hypercohomology spectra and Thomason's descent theorem, in Algebraic $K$-theory (Toronto, ON, 1996) (V. Snaith, ed.), Fields Inst. Commun. 16, pages 221-277, Amer. Math. Soc., Providence, RI, 1997.

[14] R.W. Thomason, Algebraic K-theory and étale cohomology, Ann. Sci. École Norm. Sup. (4) 18 (1985), 437-552.

[15] J.S. Wilson, Profinite groups, The Clarendon Press, Oxford University Press, New York, 1998.

Daniel G. Davis dgdavis@louisiana.edu

University of Louisiana, Mathematics Department, 1403 Johnston Street, Maxim Doucet Hall, Room 217, Lafayette, LA, 70504, USA 